\title{
SOLVING HEAT CONDUCTION PROBLEMS IN MOVABLE BOUNDARY DOMAINS UNDER INTENSIVE PHYSICAL- CHEMICAL TRANSFORMATION CONDITIONS
}

\author{
A.N. Garashchenko ${ }^{1, a}$, V.P. Rudzinsky ${ }^{2}$, and N.A. Garashchenko ${ }^{3}$ \\ ${ }^{1}$ Central Research Institute of Special Machinery, JSC, Khotkovo, Moscow Region, Russia \\ ${ }^{2}$ National Research Tomsk Polytechnic University, Tomsk, Russia \\ ${ }^{3}$ Teplozashchita LLC, Sergiev Posad, Moscow Region, Russia
}

\begin{abstract}
Results of solving problems of simulating temperature fields in domains with movable boundaries of characteristic zones of intensive physical-chemical and thermomechanical transformations to be realized in materials upon high-temperature heating have been presented. Intumescent fire-protective coatings based on organic and mineral materials are the object of study. Features of numerical realization of input equation systems taking into account, in particular, a dynamics of considerable increase and subsequent decrease of the intumescent layer thickness have been considered. Example calculations for structures of metal and wood protected with various coatings are given. Results of calculating twodimensional temperature fields in polymer composite square-shaped structures with internal cruciform load-bearing elements have been presented. The intumescent coating is arranged on the external surface of a structure. The solution of the above-listed problems is of important significance to provide fire protection of different-purpose structures and products.
\end{abstract}

Problems of simulating temperature fields in materials suffering physical-chemical and thermalmechanical transformations upon heating under the action of high-temperature gas medium fall into a number of important heat-exchange problems. Such materials include, for example, intumescent fireprotective coatings designed for fire protection of different-purpose structures and products of metals, wood and polymer composite materials.

Experiments for structures with fire protection, when simulating real fire conditions, are laborconsuming and high-cost. In many cases, moreover, they are accompanied with difficulty solved physical-similarity problems. In this connection, a mathematical simulation of complex processes under investigation is expedient. When developing corresponding techniques and calculation programs, it is necessary to take into consideration that heating of materials under study is accompanied with a boundary movement of observable zones of physical-chemical and thermalmechanical transformations. The simulation should be carried out taking into account the formation processes and change dynamics of the thickness of the swollen material layer that, in essence, is the protective layer of the surface of some object under fire conditions. Till the present time, a general

\footnotetext{
${ }^{\mathrm{a}}$ Corresponding author: a.n.gar@mail.ru
} 
theory of heat transfer processes under intensive physical-chemical transformation conditions in domains with movable boundaries has not been developed, therefore these studies remain urgent.

Most of fire-protective coatings has an organic base and when simulating their warm-up it is necessary to take into account that, on a level with corresponding physical-chemical processes (thermal decomposition, pore-formation, filtering and others), the account of intensive intumescence whose multiplicity for different materials can be varied within the limits from 20 to 120 is the most important. Special experiments have shown that such materials have a feature to reduce the thickness of formed swollen layer (coke foam) in time. It occurs at the expense of burning out carbon that is contained in the layer as well as because of the coke-foam "compression" as the quantity of forming gaseous thermal-decomposition products, passing through it, is reduced. The surface to be heated is the most important movable boundary of a material besides which in the simulation process it is also necessary to account the movement of boundaries of the thermal decomposition zone and the material plastic state zone within which the material thickness (intumescence) is increased multiply.

A mathematical model of the process under investigation is a system of heat conduction equations, Darcy's form motion, continuity (for thermal decomposition products), boundary motion equations and state equations with corresponding boundary conditions. The original equation system for this kind of materials is presented in $[1,2]$.

Also, the fire-protective mineral-based coatings are applied. They have lower intumescence multiplicity (up to 10). At the same time, together with thermal decomposition of material upon hightemperature heating, the complex of accompanying processes (moisture evaporation, vapor-gas mix filtration, deposition and subsequent secondary-moisture condensation) takes place. For these materials, it is necessary additionally to take into account the movement of evaporation boundary of moisture contained in porous structure as well as the movement of boundaries of the secondary condensate zone. The system of heat transfer equations for this kind of materials is presented in [3].

Solving the intumescent organic-based material warm-up problem was accompanied with the development of a special method and an initial equation system numerical-realization algorithm. Solving the formulated boundary problem has conducted by a finite-difference method. A differenceequation system for the movable boundary domain was solved by an iteration method for each temporal ply. To account a change in computational domain sizes, irregular difference grids with variable temporary mesh width and space-grid point number were used.

The iteration method modification [2], providing the iteration process convergence on the base of general principles and algorithms in the presence of all above-listed physical-chemical processes, deforming the computational domain and features of heat-mass transfer in it has been realized.

The iteration-averaging procedure has been employed. Apart from providing the iteration process convergence, it was also allowed the second accuracy order to be obtained both when linearizing boundary conditions and when approximating the whole difference algorithm. The boundary position of the decomposition and intumescence zones was determined using a field of temperatures in a material by means of the explicit interpolation selection of coordinates corresponding to temperatures of destruction onset, plasticity onset, destruction end, plasticity end and other. The introduction of such procedure caused, in some cases, the reduction of the iteration convergence rate but excluded the iteration process divergence possibility.

The necessity to take into account the essential unsteady deformation intensively running in enough small thickness in comparison with the general thickness of swollen material caused the use of nonuniform and irregular difference grid for numerical analysis. A discrepancy between characteristic dimensions of the immediate intumescence zone and the warm-up zone has been predetermined the necessity of building a difference grids with $8 \ldots 10$-fold clustering (increasing of point number) in the intumescence zone, in comparison with the initial material domain, with subsequent the clustering relaxation as the going out beyond the boundary of the plasticity zone occurs.

Numerical investigations showed that to provide conditions of stability and convergence of the difference solution with the corresponding approximation order in the case under consideration, a formal control of grid parameters is deficient. To carry out these conditions, special iteration-process control procedures regulating ties between grid parameters, proceeding from the iteration convergence 
rate in the appointed time segment, were developed. Moreover, the choice of a numerical value of the iteration convergence criterion was fulfilled in this way so that subsequent decreasing the difference grid mesh-width size did not practically influence the temperature-field calculation results.

Features of numerical solution of the heat-conduction problem for a plate of water-containing mineral-based materials are bound, on the whole, with the presence of two movable fronts (vapors of adsorbed moisture and condensation) as well as the dehydration zone, in which the intensive local energy release (during condensation) or energy absorption (during evaporation and dehydration) is realized. The presence of these fronts and the zone (three nonlinear heat absorption and release sources) essentially complicates the numerical solution process of the formulated problem. In this connection, the irregular and nonuniform difference grid whose parameters were selected from conditions of providing convergence and stability of this difference solution was used.

The reconstruction of the difference grid was performed for each mesh width in time. With this, the grid clustering (increasing of point number per physical length unit) in the vicinity of sources or sinks, and the rarefaction on segments through which heat-realize or heat-absorption fronts have passed, were carried out. The total number of points on 3D coordinate for typical heat-transfer conditions was, with this, not less than 300. Procedures for matching schemes of discretizing the solution domain with a temperature field on the preceding iteration have been developed. For numerical realization of the mathematical model of the water-containing intumescent fire protection, the listed techniques were combined with techniques used for the intumescent organic-based fire protection.

Typical results of the numerical simulation for some intumescent coatings designed to protect steel and wood structures are presented in Figures 1,2 as an example. Figure 1 shows results of experimental investigations for steel structures with reduced metal thickness to be determined as structural cross-section area-to-perimeter ratio, equal to $3.4 \mathrm{~mm}$. One of structures was tested with the Protherm Steel organic-based intumescent coating applied on it, that has 45 -fold intumescence and the intumescence-onset temperature about $220^{\circ} \mathrm{C}$ (Figure 1a). Other structure had the OSP-1 mineralbased coating with 7-fold intumescence (Figure $1 \mathrm{~b}$ ).

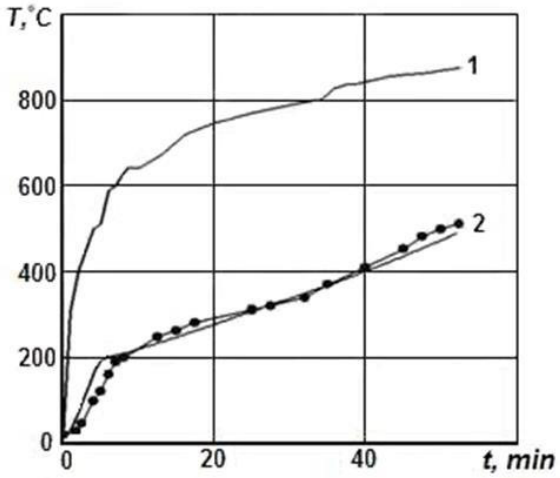

a)

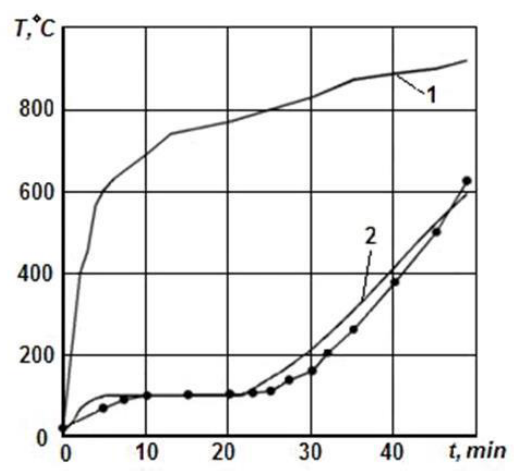

b)

Figure 1. Temperatures of gas medium (1) and steel columns with reduced metal thickness of $3.4 \mathrm{~mm}(2)$ with Protherm Steel coating $1.06 \mathrm{~mm}$ thick (a) and OSP-1coating $2.0 \mathrm{~mm}$ thick (b) as a function of time: $\longrightarrow$ - experiment; —— calculation.

A complex of material properties necessary for the numerical simulation, specifically, the intumescence multiplicity, was determined using the radiant heating unit. Heating was carried out with measurement of real gas-medium temperatures which were used in calculations. The comparison, an example of which is presented in Figure 1, has demonstrated close agreement of computational results with experimental data. Calculated curves have represented a behavior of the materials.

The calculation-to-experiment comparison for intumescent coatings on wood structures (Figure 2) can be considered as an example of successful solution of the heat conduction problem for materials having the maximum intumescence. The figure shows results of experiments with glued wood panels $100 \mathrm{~mm}$ thick with Protherm Wood and Phenix DP coatings applied on them (120-fold intumescence 
and low intumescence-onset temperature about $130^{\circ} \mathrm{C}$ ). For experimental determination of the swollen layer thickness change dynamics, a special device allowing measurements to be conducted directly in the course of experiments was used. Temperatures obtained experimentally and theoretically in thermocouple points (on panel-coating boundary and along thickness of panel) are compared in Figure 2 and the swollen layer thickness change data are compared in Figure 3. Matching has demonstrated close agreement of calculation results with experimental data both in temperatures and in intumescent layer thicknesses. The main feature of these coatings, apart from maximum increase of material thickness, consists in considerable reduction of swollen layer thickness in time.

The problem of heat conduction in cylindrical structures of polymer composite materials with internal cross-shaped load-bearing elements, surface of which has the applied intumescent coating, has been also solved. These materials can be used for making columns, struts, masts and others. Apart from complicating the problem in connection with change-over from one-dimensional statement to two-dimensional one, a specific difficulty in the numerical solution was in describing the computational domain. In this case, the problem was solved in cylindrical coordinates.

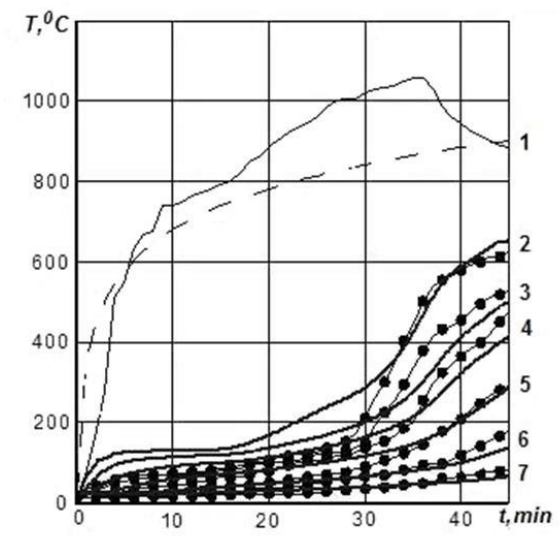

a)

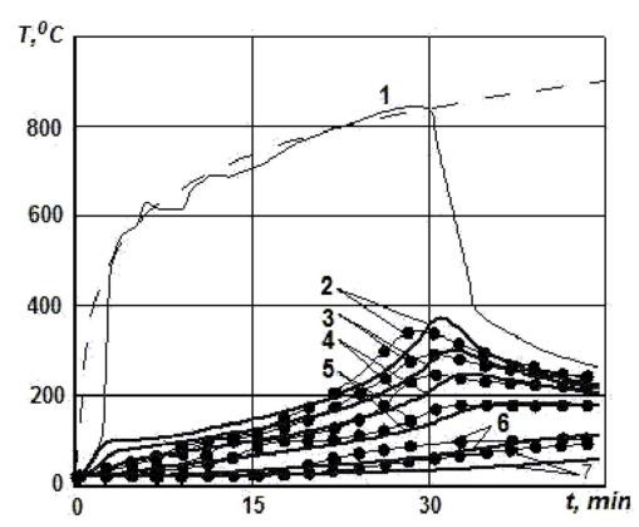

b)

Figure 2. Temperatures of gas medium (1) and on boundary between wood-glued panel and coatings (2) Protherm Wood $1.1 \mathrm{~mm}$ thick (a) and Phenix DP $0.6 \mathrm{~mm}$ thick (b), and also on distance of $2 \mathrm{~mm}$ (3), $\mathrm{mm}$ (4), $10 \mathrm{~mm}$ (5), $20 \mathrm{~mm}$ (6), $30 \mathrm{~mm}$ (7) from panel surface, as a function of time: $\_\bullet$ experiment; calculation.

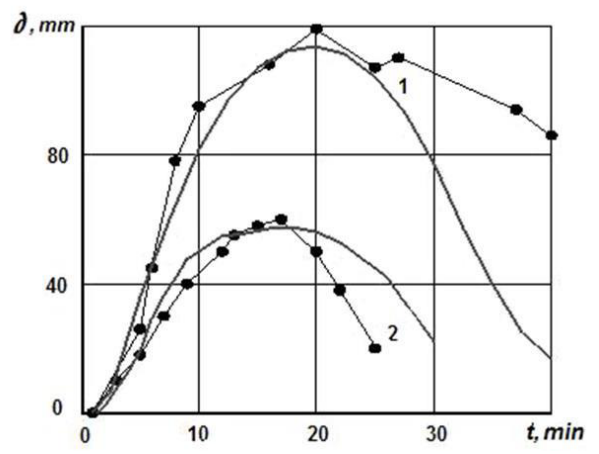

Figure 3. Swollen layer thickness-time curves obtained in experiments with wood-glued panel coated with Protherm Wood $1.1 \mathrm{~mm}$ thick (1) and Phenix DP 0.6 mm thick (2).

The square-shaped structure whose scheme is presented in Figure 4 is one of statement variants. The problem was solved in the Cartesian coordinates that required the corresponding description of the computational domain. Typical results of simulating temperature fields in the form of relationships 
for temperature in check points are presented in Figures 5, 6. Two variants were investigated: without filling inner cavities with low-density mineral-wool or basalt-fiber material and with filling the cavities with ones. The results (Figures 5, 6) show how a level of warming-up the internal loadbearing elements of a structure has been reduced when filling the cavities.

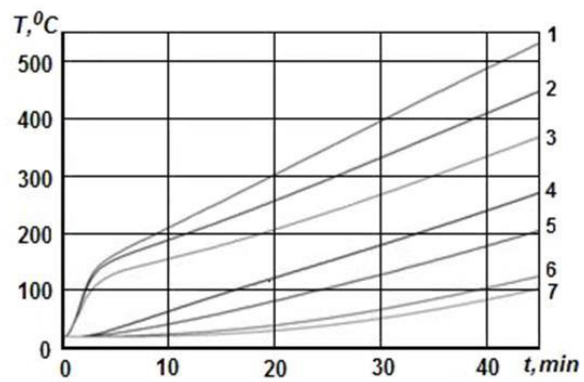

a)

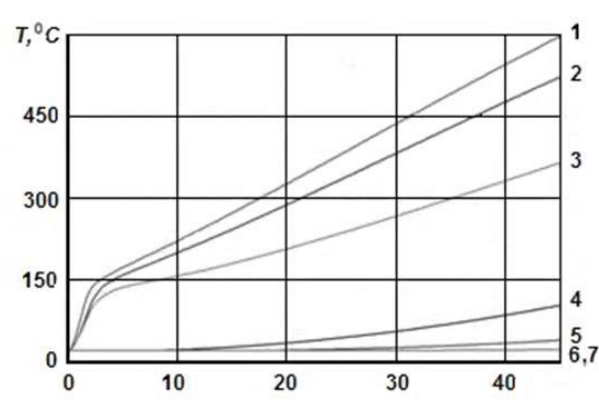

b)

Figure 5. Change in temperature with time for structures with SGK-2 coating $2 \mathrm{~mm}$ thick in check points indicated in Figure 4 in the absence (a) and in the presence (b) of filling inner cavities.

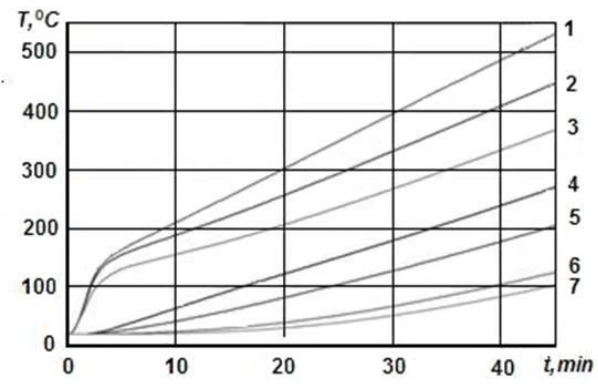

a)

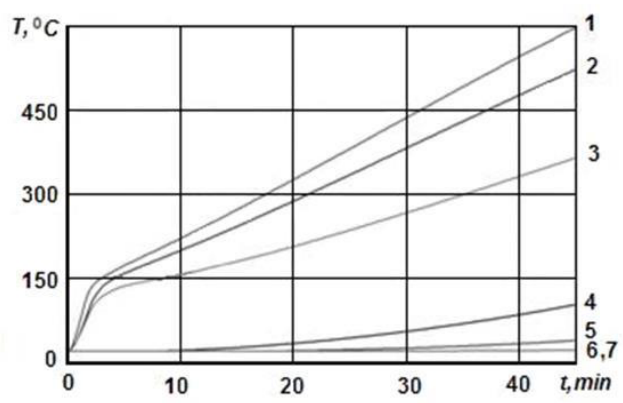

b)

Figure 6. Change in temperature with time in check points of a structure with SGK-2 coating $1 \mathrm{~mm}$ (a) and $3 \mathrm{~mm}$ (b) thick.

The results of solving the heat conduction problem group in movable boundary domains under intensive physical-chemical and thermal-mechanical transformation conditions upon heating have shown the high trustworthiness of obtained theoretical outcomes when comparing with experiments. The results obtained are also of important practical significance while creating systems and technologies of fire protection for structures and various-purpose products.

\section{References}

1. V.L. Strakhov, A.N. Garashchenko, G.V. Kuznetsov, V.P. Rudzinsky, Mathematical Modeling, 12 (5), 107 (2000)

2. V.L. Strakhov, A.N. Garashchenko, G.V. Kuznetsov, V.P. Rudzinsky, Combustion, Explosion, and Shock Waves, 37 (2), 212 (2001)

3. V.L. Strakhov, A.N. Garashchenko, G.V. Kuznetsov, V.P. Rudzinsky, High Temperature, 38 (6), 921 (2000) 